\title{
Estudo fitoquímico e avaliação in vitro da atividade anti-Trypanosoma cruzi cepa $Y$ de Pilocarpus spicatus St. Hil. (Rutaceae)
}

SILVA, C.V. ${ }^{*} ;$ BOMFIM, F.C.C. ${ }^{2 ;}$ DOS SANTOS, M.A.V. ${ }^{2}$; VELOZO, E.S. ${ }^{1}$

${ }^{1}$ Departamento do Medicamento, Faculdade de Farmácia, Universidade Federal da Bahia, Rua Barão de

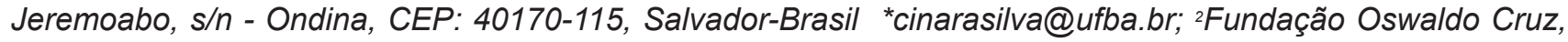
Centro de Pesquisas Gonçalo Moniz, Rua Waldemar Falcão, 121, Candeal, CEP: 40296-710, Salvador-Brasil.

\begin{abstract}
RESUMO: A investigação química da espécie Pilocarpus spicatus, popularmente conhecida como jaborandi e usada na medicina tradicional para doenças como estomatite, febre, bronquite e psoríase, teve por objetivo o isolamento e/ou identificação de substâncias ativas e a avaliação da atividade antiparasitária dos extratos frente às formas epimastigotas de Trypanosoma cruzi. O estudo resultou na identificação de nove substâncias, tais como: tridecanona, 2-heptadecanona, espatulenol, aromadendreno, $\beta$-cariofileno, ácido 3a-hidroxitirucala-7,24dien-21-óico, (+)-isoangenomalina, episesamina e sesamina. As estr uturas dos compostos foram elucidadas por análises espectroscópicas e comparação com dados da literatura. Os extratos hexânico e metanólico de folhas e raízes foram testados in vitro contra o Trypanosoma cruzi cepa $Y$ e apresentaram atividade tripanomicida.
\end{abstract}

Palavras-chave: Rutaceae, Pilocarpus spicatus, atividade tripanomicida

\begin{abstract}
Phytochemical study and in vitro evaluation of the anti-Trypanosoma cruzi Y strain activity of Pilocarpus spicatus A. St. Hil. The chemical investigation of the species Pilocarpus spicatus - popularly known as jaborandi and used in traditional medicine for diseases, such as stomatitis, fever, bronchitis and psoriasis - aimed to isolate and / or identify the active substances and evaluate the antiparasitic activity of the extracts against the Trypanosoma cruzi epimastigote forms. The study resulted in the identification of nine substances, such as tridecanone, 2-heptadecanone, spathulenol, aromadendrene, $\beta$-caryophyllene, 3a-hydroxytirucalla-7,24-dien-21-oic acid, (+)-isoangenomaline, episesamin and sesamin. The structures were elucidated by spectroscopic analysis and comparison with literature data. The hexane and methanol extracts from leaves and roots were tested in vitro against Trypanosoma cruzi $Y$ strain and showed trypanocidal activity.
\end{abstract}

Keywords: Rutaceae; Pilocarpus spicatus; trypanocidal activity.

\section{INTRODUÇÃO}

Pilocarpus Vahl é um gênero neotropical de plantas arbustivas e arbóreas, com distribuição geográfica que se estende desde o sul do México e América Central - incluindo as Pequenas e Grandes Antilhas - até o sul da América do Sul. É composto por 17 espécies e 14 são encontradas exclusivamente no território brasileiro (Skorupa,1996; Skorupa e Pirani, 2004; Sawaya et al., 2011).

Algumas espécies de Pilocarpus são chamadas popularmente como jaborandi, nome derivado da língua tupi-guarani, que significa planta que causa saliva. Este gênero é constantemente estudado devido às propriedades farmacológicas como potente estimulante de secreção e diurético (Corrêa, 1969). As folhas de Pilocarpus são usadas em infusões para o tratamento de febre, estomatite, bronquite e psoríase (Holmsted et al., 1979).

$\mathrm{O}$ isolamento da pilocarpina, um alcaloide imidazólico, em 1875 e o uso na oftalmologia para glaucoma mostram a importância desta planta da flora brasileira (Pinheiro, 1997).

Além dos alcaloides, um grande número de metabólitos secundários é reportado no gênero Pilocarpus, tais como: as cumarinas (Amaro-Luis et al., 1990), os flavonoides (Bertrand et al., 2001), os hidrocarbonetos oxidados (Negri et al., 1998), os 
terpenos (Andrade Neto et al.,1994), e as lignanas (Souza et al., 2003).

Neste gênero, destaca-se Pilocarpus spicatus, espécie encontrada apenas no Brasil, nos estados de São Paulo, Rio de Janeiro, Espírito Santo e Bahia. O estudo fitoquímico da espécie demonstrou a presença da cumarina chalepina, a qual apresentou atividade tripanomicida, além de vários terpenos, tais como: $\alpha$ e $\beta$-pineno, canfeno, Z,Z-farnesol, E-nerolidol e allo-aromadendreno (Andrade et al. 1994; 2002; Pavão et al., 2002; Santos e Moreno, 2004).

As atividades antibacterianas e imunomodulatórias do extrato de folhas desta espécie foram estudadas, bem como, a atividade dos extratos brutos de caule de $P$. spicatus e suas frações, frente à forma tripomastigota de $T$. cruzi com resultados superiores a $80 \%$ de inibição do parasita (Costa et al., 2009; Mafezoli et al., 2000).

Sabe-se que a composição química de espécimes de uma mesma espécie varia a depender das condições geográficas do local de coleta, bem como da parte estudada. Por este motivo, o presente trabalho teve por objetivo o isolamento, a identificação de substâncias ativas de um espécime de Pilocarpus spicatus coletado no semiárido baiano e a avaliação da atividade antiparasitária dos extratos frente à forma epimastigota do Trypanossoma cruzi cepa Y, agente etiológico da Doença de Chagas.

\section{MATERIAL E MÉTODO}

O espécime de Pilocarpus spicatus A. St. Hil. estudado foi coletado nas margens da estrada que liga o município de Piatã ao distrito Inúbia, Chapada Diamantina - BA, em 26 de Março de 2005. A identificação botânica foi realizada pela Profa Maria Lenise da Silva Guedes, curadora do Herbário Alexandre Leal Costa (ALCB), no Instituto de Biologia da Universidade Federal da Bahia e uma exsicata encontra-se catalogada no ALCB, sob o número 69167.

As raízes $(1,40 \mathrm{Kg})$, caule $(1,20 \mathrm{Kg})$ e folhas $(186,32 \mathrm{~g})$ de Pilocarpus spicatus foram secos, triturados e submetidos às extrações sucessivas com $n$-hexano e metanol ( $3 \times 3,0 \mathrm{~L} /$ solvente) em temperatura ambiente. $\mathrm{O}$ caule seco foi moído e macerado à frio com $n$-hexano e metanol por três vezes.

As folhas foram secas ao ar livre, trituradas e extraídas por três vezes com $n$-hexano e metanol $(\mathrm{MeOH})$. Todos os extratos foram concentrados sob pressão reduzida.

O extrato hexânico das raízes foi separado por coluna cromatográfica filtrante, empacotada a seco com gel de sílica 60 , utilizando-se como fase móvel misturas de diclorometano (DCM), acetato de etila (AcOEt) e $\mathrm{MeOH}$ em gradiente de polaridade crescente ( $n$-hexano puro, $n$-hexano-AcOEt nas proporções: 99:1, 9:1, 8:2, 7:3 e 1:1, além de $\mathrm{MeOH}$ puro), obtendo-se 7 frações de aproximadamente $200 \mathrm{~mL}$ cada. Após a evaporação dos solventes, todas as frações desta coluna foram submetidas à cromatografia em camada delgada comparativa (CCDC), analisadas e agrupadas em 06 frações.

A fração 01 da coluna filtrante $(6,23 \mathrm{~g})$ apresentou-se como um óleo de coloração amarela e, quando analisada em cromatografia em camada delgada (CCD) e revelada na luz ultravioleta (UV) e em vapores de iodo apresentou uma única mancha, a qual, em seguida, foi submetida à cromatografia gasosa acoplada à espectrometria de massa (CG/ EM), sendo possível a identificação da mistura das substâncias 1, 2, 3, 4 e 5 .

A fração $02(6,85 \mathrm{~g})$ foi fracionada por coluna cromatográfica de sílica gel 60 , utilizando como eluentes, misturas de $n$-hexano e acetato de etila em gradiente crescente de polaridade a começar com n-hexano puro e terminar com AcOEt puro. Foram obtidas 18 frações, que depois de analisadas por CCD, resultaram em 8 frações. Na fração 3 desta coluna foi determinada a substância 6 .

A fração $04(5,65 \mathrm{~g})$ foi purificada por cromatografia em coluna (CC), utilizando como fase móvel solventes em ordem crescente de polaridade: $n$-hexano, $\mathrm{DCM}$ e $\mathrm{MeOH}$. Este procedimento levou à obtenção de 40 frações, que foram reunidas em 9 frações, após análise com CCD. Da fração 04 desta coluna foi identificada a substância 7 .

O extrato hexânico de caule, com massa de $31,20 \mathrm{~g}$, foi separado em coluna de sílica gel 60 , eluído com misturas de clorofórmio $\left(\mathrm{CHCl}_{3}\right)$ e $\mathrm{MeOH}$ nas concentrações: $\mathrm{CHCl}_{3}$ puro, $\mathrm{CHCl}_{3}-$ $\mathrm{MeOH}$ - 99:1, 98:2, 97:3, 95:5, 9:1; 85:15, 7:3, 6:4, $1: 1,4: 6,3: 7,2: 8,15: 85,1: 9, \mathrm{MeOH}$ puro; resultando em um total de 16 frações. Destas, a fração 3 $(1,13 \mathrm{~g})$ foi usada para separação cromatográfica, utilizando misturas de $n$-hexano e DCM em gradiente crescente de polaridade, desta coluna foram obtidas 32 frações separadas a partir do acompanhamento da coluna com luz ultravioleta. Da fração 07 desta coluna $(56,12 \mathrm{mg})$ foi isolada a substância 8 .

Com o objetivo de isolar alcaloides, o extrato hexânico de folhas $(15 \mathrm{~g})$ foi tratado com uma solução de $\mathrm{KOH} \mathrm{5 \%} \mathrm{(p/v)} \mathrm{e} \mathrm{extraído} \mathrm{com}$ diclorometano. Esta fração resultou em resíduo de $4,50 \mathrm{~g}$, que foi submetido a coluna isocrática com clorofórmio/metanol (99:1) fornecendo 19 frações. A fração 01 obtida nesta coluna foi separada por uma CC em sílica gel 60 , eluída com misturas de clorofórmio e acetato de etila em gradiente crescente de polaridade (n-hexano; 99:1, 98:2, 95:5, 9:1, 8:2, 7:3: 6:4, 1:1 e AcOEt), onde foram obtidas 10 frações. A fração 6 desta coluna foi identificada como 
o composto 9 .

Os ensaios Anti-Trypanosoma cruzi foram realizados no Laboratório de Biomorfologia Parasitária (LBP) do Centro de Pesquisa Gonçalo Moniz (Fiocruz). A cultura de formas epimastigotas de Trypanosoma cruzi cepa $Y$ foi mantida em meio LIT (Liver Infusion Tryptose) suplementado com $10 \%$ de soro bovino fetal (SBF), numa temperatura de $28^{\circ} \mathrm{C}$. Uma concentração inicial de $5 \times 10^{\circ}$ de parasitas $/ \mathrm{mL}$ foi utilizada e incubada em placas de 24 poços, utilizando-se $40 \mu \mathrm{L}$ de uma solução dos extratos com dimetilsulfóxido (DMSO) na concentração de $10 \mathrm{mg} \mathrm{mL}^{-1} \mathrm{e}$ completando com 2 $\mathrm{mL}$ de meio/poço.

Os experimentos foram realizados em triplicata e para o controle do experimento foi utilizado DMSO e meio de cultura. O número de parasitas foi apurado após 96 horas de tratamento, por contagem em hemocitômetro de Neubauer.

Os espectros de ressonância magnética nuclear (RMN) de ${ }^{1} \mathrm{H}(300 \mathrm{MHz}),{ }^{13} \mathrm{C}(75 \mathrm{MHz}) \mathrm{e}$ de melhoramento sem distorção por transferência de polarização a $135^{\circ}$ (DEPT $135^{\circ}$ ) foram obtidos nos espectrômetros Varian Gemini 300 e Mercuri 300. Os cromatogramas foram revelados através de irradiação de lâmpada U.V. nos comprimentos de onda de $254 \mathrm{~nm}$ e $366 \mathrm{~nm}$, vapores de iodo e reveladores químicos como Dragendorff, LiebermanBurchard e solução de $\mathrm{FeCl}_{3}$. Nas separações cromatográficas em coluna, foram usadas sílica gel 60 (70-230 mesh/ 0,063-0,200 mm/ 40-63 mm) das marcas Merck e Vetec. Os espectros de massas foram registrados por impacto eletrônico (70 eV) em CG-EM de marca Shimadzu, modelo GC-17A equipado com injetor e divisor de fluxo e partição da amostra de 1:50 e coluna capilar $(30 \mathrm{~m} \times 0,25$ $\mathrm{mm} \times 0,1 \mathrm{~mm}$ ) e Tinj $60^{\circ} \mathrm{C}$ com elevação de $3^{\circ} \mathrm{C}$ $\mathrm{min}^{-1}$ até $200^{\circ} \mathrm{C}$ e depois $10^{\circ} \mathrm{C} / \mathrm{min}$ até $280^{\circ} \mathrm{C} \mathrm{com}$ tempo total de análise de $100 \mathrm{~min}$. A quantificação dos constituintes foi obtida por integração eletrônica pela técnica de normalização. Na análise qualitativa, se utilizou as mesmas condições e, como detector, o espectrômetro de massas GC/MS - QP5000 (Shimadzu).

\section{RESULTADO E DISCUSSÃO}

Uma alíquota da fração 01 , originada da coluna filtrante do extrato hexânico de raiz, foi injetada em cromatógrafo à gás acoplado à espectrometria de massa (CG/EM) nas condições de Adams. Após a determinação dos índices de retenção (IK) e comparação dos espectros de massa com dados da literatura foi possível a identificação dos sesquiterpenos ß-cariofileno (1) IK1409, aromadendreno (2) - IK1441, espatulenol (3) - IK1484 e dos hidrocarbonetos oxidados 2-tridecanona (4) - IK1578 e 2-heptadecanona (5) - IK1700 (Adams, 1995).

A segunda fração da coluna filtrante do extrato hexânico de raiz forneceu $56,12 \mathrm{mg}$ de um sólido branco amorfo. $\mathrm{O}$ espectro de $\mathrm{RMN}{ }^{1} \mathrm{H}$ desta substância apresentou sete simpletos em $\delta$ 1,$67 ; \delta 1,58 ; \delta 0,95 ; \delta 0,93 ; \delta 0,91 ; \delta 0,88$ e $\delta 0,72$, indicando a presença de sete grupos metílicos na molécula e sugerindo tratar-se de triterpeno. $\mathrm{O}$ espectro de $\mathrm{RMN}{ }^{1} \mathrm{H}$ apresentou ainda um simpleto largo em $\delta 3,50$ atribuído ao $\mathrm{H}-3$, em axial. $\mathrm{O}$ dupleto largo em $\delta 5,23(J=3,3 \mathrm{~Hz})$ e o tripleto em $\delta 5,08$ indicam a presença de próton vinílico em ligação dupla endo-cíclica $\Delta^{7}$, além de uma ligação dupla na cadeia lateral $\Delta^{24}$, apontando para esqueleto do tipo tirucalano.

O espectro de RMN de ${ }^{13} \mathrm{C}$ desta substância apresentou 30 sinais de carbonos, confirmando a estrutura triterpênica. Além do sinal em $\delta$ 181,65, referente ao carbono carboxílico, foram encontrados: sinais em $\delta 118,13$ e $\delta 145,62$ relativos aos carbonos olefínicos da insaturação entre carbonos C-7 e $\mathrm{C}-8$, sinais em $\delta 132,09$ e $\delta 123,65$ dos carbonos olefínicos C-25 e C-24 da cadeia lateral e o sinal em $\delta 76,47$, atribuído ao carbono carbinólico, com deslocamento característico de $\mathrm{OH}$ na posição equatorial. Através do experimento DEPT-135 foi possível confirmar a presença de 8 carbonos metínicos, 9 carbonos metilênicos e 7 metílicos. Estes dados comparados com aqueles fornecidos pela literatura identificam esta substância como ácido 3a-hidroxi-tirucala-7,24-dien-21-óico (Mora et al., 2001).

A fração 04 foi submetida à purificação por cromatografia em coluna clássica (C.C) com sílica gel, utilizando como fase móvel misturas de hexano, diclorometano e metanol, em ordem crescente de polaridade. Este procedimento forneceu 9,0 mg de um sólido branco. Os dados dos espectros de RMN de ${ }^{1} \mathrm{H},{ }^{13} \mathrm{C}$ apresentados na Tabela 1 e o espectro de correlação homonuclear de $\mathrm{H}^{1-} \mathrm{H}^{1}$ (COSY), que confirmou as multiplicidades observadas no espectro de $\mathrm{RMN}$ de $\mathrm{H}^{1}$ (Figura 1), foram comparados com a literatura (Yamaguchi et al., 1989) e possibilitaram

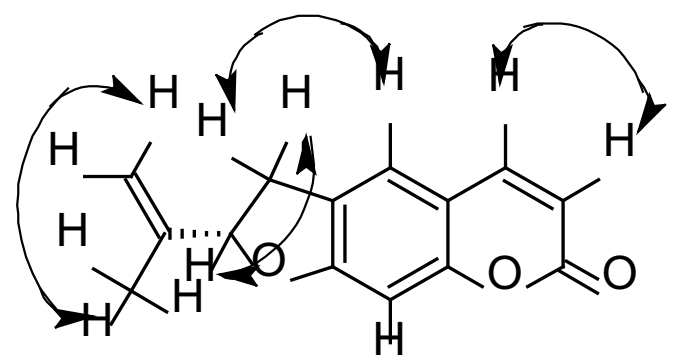

FIGURA 1. Correlações $\mathrm{H}^{1-} \mathrm{H}^{1}$ da cumarina isoangenomalina (7). 
TABELA 1. Dados espectroscópicos de $\mathrm{RMN}{ }^{1} \mathrm{H}$ e ${ }^{13} \mathrm{C}$ da cumarina (+)-isoangenomalina

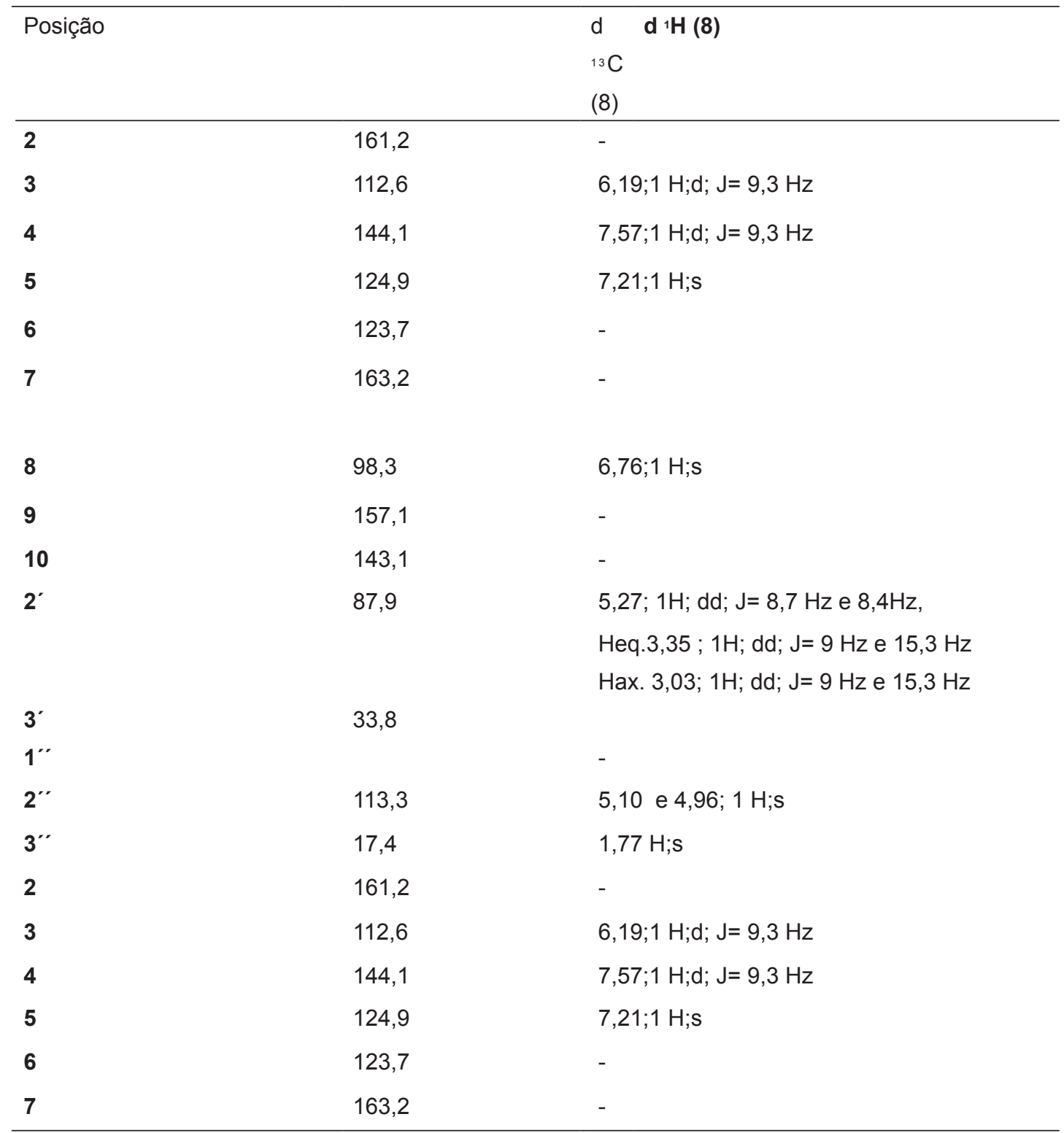

a identificação da cumarina(+) - isoangenomalina.

Após o fracionamento por cromatografia em coluna, o extrato hexânico de caule forneceu 15,20 mg de uma substância 8 e foi identificada por RMN ${ }^{1} \mathrm{H},{ }^{13} \mathrm{C}$ e DEPT-135 e dados da literatura (Hsieh et al., 2005) como a lignana sesamina, já isolada de outras espécies de Rutaceae (Tabela 2).

Uma massa de $36,0 \mathrm{mg}$ da substância 9 foi obtida do extrato hexânico de folhas, após fracionamento. A análise dos dados de $\mathrm{RMN}{ }^{13} \mathrm{C}$ e ${ }^{1} \mathrm{H}$ e comparação destes com a literatura (Brown et al., 2001) permitiu a identificação da episesamina (Tabela 2).

Os testes de atividade anti- $T$. cruzi foram realizados com extrato hexânico e metanólico das folhas, e com o extrato metanólico das raízes. As amostras apresentaram atividade tripanomicida (Figura 2). Todos os extratos apresentaram inibição maior que $50 \%$, sendo que o extrato hexânico das folhas inibiu $79 \%$, o extrato metanólico das folhas inibiu $56 \%$ e o extrato metanólico das raízes inibiu $62 \%$ do crescimento de parasitas.

Em trabalho anterior, os extratos brutos de $P$. spicatus apresentaram inibição dos parasitas maior que suas frações, sugerindo o efeito sinérgico entre os componentes do extrato (Mafezoli et al, 2000). Apesar dos estudos, até o momento, não detectarem a presença da chalepina, piranocumarina que apresenta atividade inibitória da enzima GAPDH fundamental para o Trypanosoma cruzi (Pavão et al., 2002); a presença de outros componentes com possível ação anti- T. cruzi a exemplo de terpenos, 


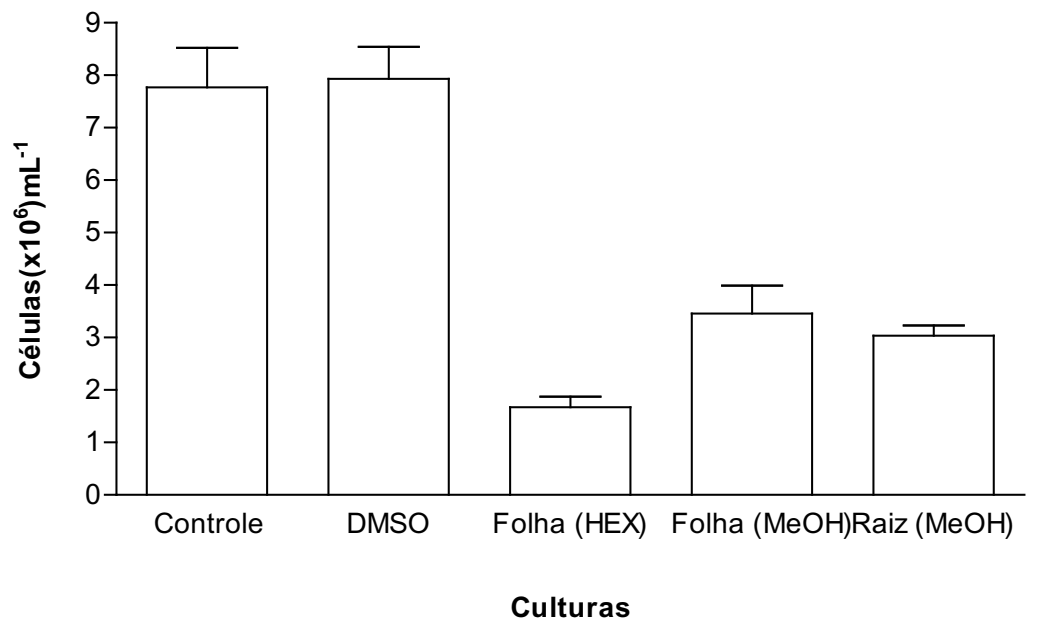

FIGURA 2. Screening dos extratos de P. spicatus frente a T. cruzi - $200 \mu \mathrm{g} / \mathrm{mL}$

TABELA 2 - Dados espectroscópicos de RMN ${ }^{1} \mathrm{H}$ e ${ }^{13} \mathrm{C}$ das lignanas sesamina (8) e episesamina (9).

\begin{tabular}{|c|c|c|c|c|}
\hline Posição & $\mathrm{d}^{\mathrm{i}} \mathrm{H}(8)$ & $d{ }^{13} C(8)$ & $d^{\prime}{ }^{\prime} H(9)$ & $\mathrm{d}^{13} \mathrm{C}(9)$ \\
\hline 1 & $3,05 \mathrm{~m}$ & 54,5 & $3,30 \mathrm{~m}$ & 54,8 \\
\hline 5 & $3,05 \mathrm{~m}$ & 54,5 & $3,30 m$ & 50,3 \\
\hline 2 & $H_{e q}: 4,23 d d$ & 71,9 & $\mathrm{H}_{\mathrm{eq}}: 3,83 \mathrm{dd} \quad(J=9,3$ e 6$)$ & 71,0 \\
\hline & $(J=9,2$ e 6,9$)$ & & $H_{a x}: 2,85 q$ & \\
\hline & $H_{a x}: 3,86 d d$ & & $(J=7,3$ e 0,9$)$ & \\
\hline 6 & $\begin{array}{c}(J=9,2 \text { e } 3,6) \\
H_{e q}: 4,23 d d\end{array}$ & 71,9 & $\mathrm{H}_{\mathrm{eq}}: 4,09 \mathrm{~d}$ & 69,8 \\
\hline & $(J=9,2$ e 6,9$)$ & & $(J=9,6)$ & \\
\hline & $\mathrm{H}_{\mathrm{ax}}: 3,86 \mathrm{dd}$ & & $\mathrm{H}_{\mathrm{ax}}: 3,86 \mathrm{~m}$ & \\
\hline 4 & $\begin{array}{l}(J=9,2 \text { e } 3,6) \\
4,71 d(J=4,2)\end{array}$ & 86,0 & $4,83 d(J=5,7)$ & 82,1 \\
\hline 8 & $4,71 \mathrm{~d}(J=4,2)$ & 86,0 & $4,39 d(J=7,2)$ & 87,8 \\
\hline 1 & - & 135,2 & - & 135,1 \\
\hline $1 "$ & - & 135,2 & - & 132,3 \\
\hline 2 & $6,85 d(J=1,2)$ & 106,7 & $6,77 \mathrm{~m}$ & 106,7 \\
\hline $2^{\prime \prime}$ & $6,85 d(J=1,2)$ & 106,7 & $6,77 \mathrm{~m}$ & 106,5 \\
\hline $3^{\prime}$ & - & 147,9 & - & 147,3 \\
\hline $3 "$ & - & 147,9 & - & 146,7 \\
\hline $4^{\prime}$ & - & 148,2 & - & 148,1 \\
\hline $4 "$ & - & 148,2 & - & 147,8 \\
\hline $5^{\prime}$ & $6,77 d(J=8,4)$ & 108,4 & $6,77 \mathrm{~m}$ & 108,2 \\
\hline $5 "$ & $6,77 d(J=8,4)$ & 108,4 & $6,77 \mathrm{~m}$ & 108,2 \\
\hline $6^{\prime}$ & $6,79 \mathrm{dd}$ & 119,5 & $6,77 \mathrm{~m}$ & 119,8 \\
\hline $6^{\prime \prime}$ & $\begin{array}{c}(J=8,4 \text { e } 1,2) \\
6,79 \mathrm{dd}\end{array}$ & 119,5 & $6,77 \mathrm{~m}$ & 118,8 \\
\hline $1^{\prime \prime}$ & $\begin{aligned} &(J=8,4 \text { e } 1,2) \\
& 5,96 \mathrm{~s}\end{aligned}$ & 101,3 & $5,95 \mathrm{~s}$ & 101,2 \\
\hline $1^{\prime \cdots}$ & $5,96 \mathrm{~s}$ & 101,3 & $5,97 \mathrm{~s}$ & 101,3 \\
\hline
\end{tabular}


<smiles>C=C1CC/C=C(/C)CC[C@@]2(C)C1CC2(C)C</smiles>

1

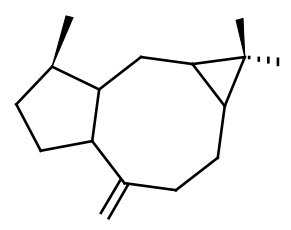

2<smiles>C=C1CCC2C(CC3C1CC[C@]3(C)O)C2(C)C</smiles>

0

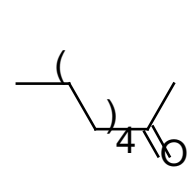

4

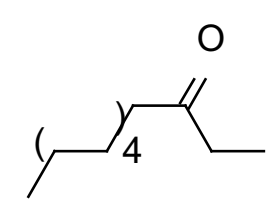

5<smiles>C=C(C)C</smiles><smiles>C=C(C)[C@@H]1Cc2cc3ccc(=O)oc3cc2O1</smiles>

7 $5^{\prime \prime}$

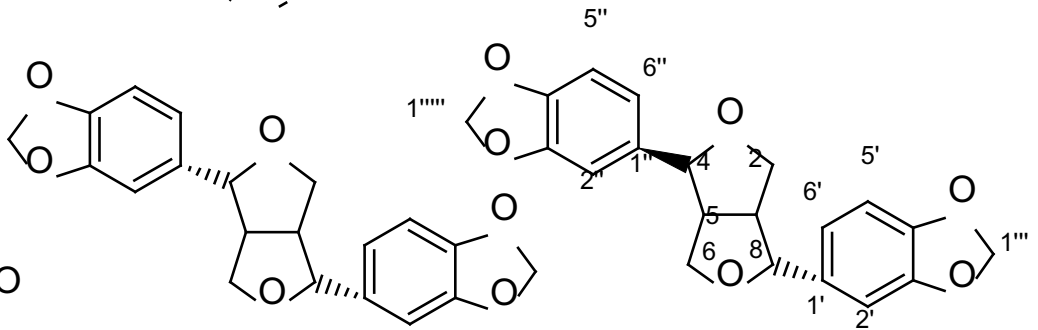

8
9

FIGURA 3. Substâncias identificadas nos extratos de caule, folhas e raiz de Pilocarpus spicatus.

cumarinas e lignanas pode explicar os resultados obtidos. Porém, estes resultados serão investigados para elucidar qual ou quais metabólitos presentes são responsáveis por tal atividade.

O presente trabalho com a espécie $P$. spicatus permitiu identificar 09 compostos: 2-tridecanona (1), 2-heptadecanona (2), espatulenol (3), aromadendreno (4), beta-cariofileno (5), o ácido 3a-hidroxi-tírucala-7,24-dien-21-óico (6) isoangenomalina (7), episesamina (8) e sesamina (9). As substâncias 6, 7, 8 e 9 ainda não haviam sido isoladas do gênero Pilocarpus, contribuindo, portanto, para a quimiotaxonomia deste gênero.

\section{AGRADECIMENTO}

Os autores agradecem a CAPES, FAPESB e CNPq pelas bolsas e apoios financeiros concedidos.

\section{REFERÊNCIA}

ADAMS, R.P. Identification of essencial oil components by gas chromatography/mass spectrometry. Illinois: Allured Publishing: Carol Stream. 1995. 469p.

AMARO-LUIS, M. et al. New coumarins from Pilocarpus goudotianus. Planta Medica, v.56, p.304-6, 1990.

ANDRADE NETO, J.M. et al. 24-methyl-25- ethyldammarane derivatives from Pilocarpus spicatus. Phytochemistry, v.35, n.3, p.739-43, 1994.

ANDRADE NETO, J.M. et al. Volatile constituents of different populations of Pilocarpus spicatus Saint Hill. (Rutaceae) from the Northeast of Brazil. The Journal of Essential Oil Research, v.14, p.319-24, 2002.

BERTRAND, C. et al. Constituents of Pilocarpus trachylophus. Fitoterapia, v.72, p.844-7, 2001.

BROWN, R.C.D. et al. C-H Insertion Approach to the Synthesis of endo,exo-Furofuranones: Synthesis of (+-)-Asarinin,(+-)-Epimagnolin A, and (+-)-Fargesin. The Journal of Organic Chemistry, n.66, p.6719-28, 2001. CORREA, M.P. Dicionário das plantas úteis do Brasil e das exóticas cultivadas. Rio de Janeiro: Imprensa Nacional, 1969, v.4, 374p.

COSTA, J.F.O. et al. Immunomodulatory and antibacterial activities of extracts from Rutaceae species. Revista Brasileira de Farmacognosia, v.20, n.4, p.502-505, 2010.

HOLMSTED, B. et al. Jaborandi: An interdisciplinary appraisal. Journal of Ethnopharmacology, v.1, p.321, 1979.

HSIEH, T.J. et al. NMR spectroscopic, mass spectroscopic, X-ray crystallographic, and theoretical studies of molecular mechanics of natural products: farformolide B and sesamin. Biophysical Chemistry, v.114, n.1, p.13-20, 2005.

MAFEZOLI, J. et al. In vitro activity of Rutaceae species against the trypomastigote form of Trypanosoma cruzi. Journal of Ethnopharmacology, v.73, p. 335-340, 2000. 
MORA, A.J. et al. 3a-hydroxy tirucalla-7,24-diene- 21-oic acid: A Triterpene from Protium Crenatum Sandwith. Acta Crystallographica, C57, p.638-40, 2001.

NEGRI, G. et al. An aromatic hydrocarbon from the foliar epicuticular wax of Pilocarpus jaborandi. Phytochemistry, v.49, n.1, p.127-9,1998.

PAVÃO F. et al. Structure of Trypanosoma cruziglycossomal glyceraldehyde-3- phosphate dehydrogenase complexed with chalepin, a natural product inhibitor, at 1.95 Angstron resolution. Febs Letters, v.520, p.13-7, 2002.

PINHEIRO, C.U.B. Jaborandi (Pilocarpus sp., Rutaceae): a wild species and its rapid transformation into a crop. Economic Botany, v.51, n.1, p.49-58, 1997.

SANTOS, A.P.; MORENO, P.R.H. Pilocarpus spp.: A survey of its chemical constituents and biological activities. Revista Brasileira de Ciências Farmacêuticas, v. 40, n. 2, 2004.

SAWAYA, A.C.H.F. et al. Screening species of Pilocarpus
(Rutaceae) as sources of pilocarpine and other imidazole alkaloids. Genetic Resources and Crop Evolution, v. 58, p.471-480, 2011.

SKORUPA, L.A. Revisão taxonômica de Pilocarpus Vahl (Rutaceae). 1996. 426p. Tese (Doutorado - Área de concentração - Botânica) - Instituto de Biologia, Universidade de São Paulo, São Paulo.

SKORUPA, L.A.; PIRANI, J.R. A new species of Pilocarpus (Rutaceae) from northern Brazil. Brittonia, v.56, p. 147-150, 2004.

SOUZA, R.C. et al. Constituintes químicos de Pilocarpus grandiflorus (Rutaceae). In: REUNIÃO ANUAL DA SOCIEDADE BRASILEIRA DE QUÍMICA, 26. Poços de Caldas, 2003. Resumos. São Paulo: SBQ, 2003, PN-203.

YAMAGUCHI, S. et al. The synthesis of some dimethylpyranocoumarins and isopropenyldihydrofuranocoumarins. Bulletin of the Chemical Society of Japan, v.62, p.3593-7, 1989. 